\title{
Fitness and eco-physiological response of a chytrid fungal parasite infecting planktonic cyanobacteria to thermal and host genotype variation
}

Ramsy Agha (1) https://orcid.org/0000-0002-6109-4624, Alina Gross, Melanie Gerphagnon (1) https://orcid.org/00000002-3363-4355, Thomas Rohrlack, Justyna Wolinska (i) https://orcid.org/0000-0003-2913-2923

DOI

$\underline{10.1017 / 50031182018000215}$

Original publication date

26 February 2018

Document version

Accepted version

Published in

Parasitology

\section{Citation (Vancouver)}

Agha R, Gross A, Gerphagnon M, Rohrlack T, Wolinska J. Fitness and eco-physiological response of a chytrid fungal parasite infecting planktonic cyanobacteria to thermal and host genotype variation. Parasitology. 2018;145(10):1279-86. 
1 Fitness and eco-physiological response of a chytrid fungal parasite infecting planktonic

2 cyanobacteria to thermal and host genotype variation

3

4 Ramsy Agha $^{1 *}$, Alina Gross ${ }^{1,2}$, Melanie Gerphagnon ${ }^{1}$, Thomas Rohrlack ${ }^{3}$ and Justyna Wolinska ${ }^{1,2}$ 5

$6 \quad{ }^{1}$ Department of Ecosystem Research, Leibniz-Institute of Freshwater Ecology and Inland

7 Fisheries (IGB), Müggelseedamm 301, Berlin, 12587, Germany

8

$9{ }^{2}$ Institute of Biology, Freie Universität Berlin, Königin-Luise-Straße 1-3, Berlin, 14195,

10 Germany.

11

$12{ }^{3}$ Faculty of Environmental Sciences and Natural Resource Management, Norwegian University

13 of Life Sciences, P.O. Box 5003, NO-1432, Ås, Norway

15 * Corresponding author: ramsyagha@gmail.com

16 Department of Ecosystem Research, Leibniz-Institute of Freshwater Ecology and Inland Fisheries

17 (IGB), Müggelseedamm 301, Berlin, 12587, Germany

18 Tel: $+49(30) 64181754$

19 Fax: $+49(0) 3064181682$

20

21

22

23

Running title: Eco-physiology of a chytrid parasite

24 
27 Understanding how individual parasite traits contribute to overall fitness, and how they are 28 modulated by both external and host environment, is crucial for predicting disease outcome. 29 Fungal (chytrid) parasites of phytoplankton are important yet poorly studied pathogens with the 30 potential to modulate the abundance and composition of phytoplankton communities and to drive 31 their evolution. Here, we studied life-history traits of a chytrid parasite infecting the planktonic, 32 bloom-forming cyanobacterium Planktothrix spp. under host genotype and thermal variation.

33 When expressing parasite fitness in terms of transmission success, disease outcome was largely 34 modulated by temperature alone. Yet, a closer examination of individual parasite traits linked to 35 different infection phases, such as (i) the establishment of the infection (i.e. intensity of infection) 36 and (ii) the exploitation of host resources (i.e. size of reproductive structures and propagules), 37 revealed differential host genotype and temperature $\times$ host genotype modulation, respectively. 38 This illustrates how parasite fitness results from the interplay of individual parasite traits that are 39 differentially controlled by host and external environment, and stresses the importance of 40 combining multiple traits to gain insights into underlying infection mechanisms. 
46 Key Findings

47

48 - Chytrid parasite fitness expressed as transmission success was controlled almost

49 exclusively by temperature

50 - Conversely, individual parasites traits were differentially modulated by cyanobacterial

51 host genotype and/or temperature

52 - Individual infection stages are differentially affected by host and external environment, 53 which ultimately determine parasite fitness

54

55 
58 Accurate measures of fitness are crucial for assessing the ecological and evolutionary trajectories 59 of host-parasite interactions. Fitness is generally defined as the average contribution of one 60 genotype or allele to the next or successive generations, compared to other genotypes or alleles 61 (Futuyma, 1986). Therefore, fitness of parasites is often expressed as their transmission success, 62 or, more formally, the number of secondary infections caused by a primary infection (May \& 63 Anderson, 1979). However, transmission-based fitness is a composite variable which integrates 64 different underlying parasite traits that individually contribute to transmission success (Antolin 2008; McCallum et al., 2017). These traits constitute fitness components that are related to specific phases of infection (e.g. infection success, host resource exploitation efficiency, reproductive output, etc.) and are often under host genotype and/or environmental control (Vale \& Little, 2009; Van den Wyngaert et al., 2014; Wolinska \& King, 2009). Unravelling how the host genotype and external environment modulate these individual traits would grant a deeper understanding of the biology of the interaction between antagonists, with regard to the mechanisms of infection, their ecophysiology, and the basis of host-parasite compatibility. Such insights allow for better predictions of disease dynamics in natural settings. This also contributes to the identification of the range of environmental conditions that delineate infection hotspots and/or environmental refuges from infection, which modulate the intensity of parasite-mediated selection on host populations, and thereby regulate host-parasite interactions in the wild (e.g. Kraaijeveld \& Godfray, 1999; Lively, 1999).

Phytoplankton represent the base of most aquatic food webs and is a major driver of global biogeochemical cycles (Falkowski, 2012). Phytoplankton can be lethally infected by parasitic 80 fungi belonging to the early diverging phylum Chytridiomycota (i.e. chytrids) (Sommer et al., 81 2012). The best known representative of this group of parasites is the species Batrachochytrium 82 dendrobatidis, which drives massive amphibian population declines worldwide (Vredenburg et 83 al., 2010). Chytrids infecting phytoplankton have been reported in the past (Braun, 1856; Canter, 84 1947; Canter \& Lund, 1948; Canter \& Lund, 1951), but they are attracting renewed interest as 85 accumulating evidence demonstrates their unexpected diversity and widespread distribution in 86 pelagic marine, brackish and freshwater habitats worldwide (Grossart et al., 2016; Hassett \& 87 Gradinger, 2016; Lefèvre et al., 2008; Lepère et al., 2008). The lifecycle of chytrids is 
characterized by a free-swimming stage in the form of flagellated zoospores that are assumed to find suitable hosts in the water column by chemotaxis (Muehlstein et al., 1988; Scholz et al., 2017). Once encysted on their host, chytrids develop a rhizoid system that penetrates the host cell wall to extract nutrients from it. As infection spreads, zoospores gradually develop into sporangia, epibiotic reproductive structures that release new zoospores upon maturation (Ibelings et al., 2004). Although often neglected, chytrid parasitism has potentially profound ecological implications (reviewed in Frenken et al., 2017). As lethal parasites, chytrids shape the structure and dynamics of phytoplankton populations and have the potential to delay or suppress algal blooms (Gerphagnon et al., 2017). Furthermore, by imposing strong selection on their hosts, chytrids can drive the evolution of phytoplankton populations (Gsell et al., 2013b). From a food web perspective, chytrids constitute a high-quality food source for zooplankton consumers. Therefore, chytrid parasitism can establish alternative trophic links in aquatic food webs and circumvent trophic bottlenecks typically imposed by the dominance of inedible or toxic phytoplankton (Agha et al., 2016; Kagami et al., 2007).

Yet, chytrid infections, including their underlying mechanisms and modulation by the environment, remain poorly characterized. Previous studies of chytrids infecting phytoplankton have been restricted for the most part to a few chytrid taxa available in culture. For instance, infections by the chytrids Zygorhyzidium planktonicum and Rhizophydium planktonicum, both parasitizing the diatom Asterionella formosa, have been shown to be modulated by abiotic factors such as light, nutrients and temperature (Bruning, 1991a; Bruning, 1991b; Bruning \& Ringelberg, 1987; Canter \& Jaworski, 1981). Moreover, in this diatom-chytrid system, temperature variation was shown to change the ranking of susceptibility among conspecific host genotypes, indicating genotype-by-environment interactions (Gsell et al., 2013a). In the present work, we focus on a related host-parasite system consisting of the chytrid Rhizophydium megarrhizum infecting cyanobacteria of the bloom-forming and toxin-producing genus Planktothrix. Previous studies have shown that $R$. megarrhizum can infect different, but not all, Planktothrix spp. strains (Sønstebø \& Rohrlack, 2011). Similarly, low temperatures have been shown to alleviate or even suppress infection, suggesting environmental control of chytridiomycosis (Rohrlack et al., 2015). However, parasite fitness in these studies was expressed as the incidence of infection (measured two days after exposure to the parasite), leaving the question open as to which specific parasite traits are modulated by environmental variation and how. Here, we assess the overall fitness (i.e. 
120 transmission-based fitness proxy) of the chytrid parasite $R$. megarrhizum across temperature and 121 host genotypic variation. Simultaneously, we examine different underlying parasite traits related 122 to ability to infect (i.e. incidence and intensity of infection) and reproductive output (i.e. sizes of 123 sporangia and zoosporic propagules), which jointly contribute to overall fitness. We aim to 124 disentangle the effects of host and external environment on these individual parasite traits and 125 thereby to contribute to a better characterization of the ecophysiology of this host-parasite system 126 and its underlying infection mechanisms.

127

128 
131 Host and parasite strains

132 The chytrid parasite strain Chy-Kol2008 was isolated in 2008 from Lake Kobotnvatet (Norway) 133 and identified as Rhizophydium megarrhizum (Sønstebø \& Rohrlack, 2011). Two host strains

134 belonging to the filamentous, bloom-forming cyanobacterial genus Planktothrix were used:

135 NIVA-CYA98 (Planktothrix rubescens, isolated from Lake Steinsfjörden (Norway) in 1982) and

136 NIVA-CYA630 (Planktothrix agardhii isolated from Lake Lyseren (Norway) in 2008). Host 137 strains were maintained in $\mathrm{Z} 8$ medium as non-axenic batch cultures under $16{ }^{\circ} \mathrm{C}$ and $15 \mu \mathrm{mol}$

138 photons $\mathrm{m}^{-2} \mathrm{~s}^{-1}$. The parasite was maintained in culture by transferring zoospore suspensions into 139 uninfected cultures of the host strain NIVA-CYA98, every two weeks.

141 Experimental setup

142 Before the start of the experiment, host strains were acclimated as semi-continuous cultures for 143 three weeks to their respective temperatures $\left(8{ }^{\circ} \mathrm{C}, 12{ }^{\circ} \mathrm{C}, 16^{\circ} \mathrm{C}\right.$ and $\left.20^{\circ} \mathrm{C}\right)$. Ten days before the 144 start of the experiment, a culture of the strain NIVA CYA98 was infected with the chytrid 145 parasite and incubated at $16{ }^{\circ} \mathrm{C}$ and $20 \mu \mathrm{mol}$ photons $\mathrm{m}^{-2} \mathrm{~s}^{-1}$. After 10 days, a purified zoospore 146 suspension was obtained by sequential filtration through sterile $10 \mu \mathrm{m}$ and $5 \mu \mathrm{m}$ nylon meshes 147 and a $3 \mu \mathrm{m}$ polycarbonate filter. The resulting filtrate was microscopically checked for the 148 absence of host filaments and zoospore density was quantified under a Nikon Ti Eclypse inverted 149 microscope using a Sedgewick Rafter chamber after fixation of a $1 \mathrm{ml}$ aliquot with acid Lugol. 150 The purified zoospore suspension was then used to infect triplicate $100 \mathrm{ml}$ aliquots of the 151 acclimated host strains NIVA-CYA98 and NIVA-CYA630 (host density 2000 filaments $\mathrm{ml}^{-1}$ ), 152 providing a final zoospore density of $750 \mathrm{ml}^{-1}$. Optical density at $750 \mathrm{~nm}$ of each host strain was 153 correlated to filament concentrations, and then used to obtain the desired initial host densities.

155 After initial infection, experimental cultures were sampled daily or, for low temperature 156 treatments, every other day, until incidence of infection in the cultures reached a clear asymptote 157 (i.e. plateau). After gently homogenizing the suspension, $1.5 \mathrm{ml}$ aliquots were collected and fixed 158 in $2 \%$ formaldehyde and stored at $4{ }^{\circ} \mathrm{C}$ from 2 to 5 weeks until analysis. Samples were used to 159 investigate four different parasite traits: incidence of infection (i.e. proportion of infected 160 filaments in the population), intensity of infection (i.e. mean number of sporangia per infected 
163 Incidence of infection was determined by the proportion of infected hosts after sequentially examining 200 cyanobacterial filaments. To compare the temporal dynamics of the incidence of infection across temperature treatments, a unit of physiological time was employed, namely degree-days. A degree-day is simply the product of 24-hour days and temperature. The use of this unit normalizes differences in host and parasite metabolic rates under different temperatures, making it possible to compare parasite fitness along temperature gradients (e.g. Mitchell et al., 2005).

Transmission-based fitness was determined after the formulation of May and Anderson (1983):

$$
\mathrm{R}_{0}=\beta \mathrm{N} / \alpha+b+v
$$

where $R_{0}$ is the number of infections caused by a single primary infection, $\beta$ is the parasite transmission rate, $\mathrm{N}$ is the density of hosts (kept constant in our experiment), $\alpha$ is the parasite virulence (equal to 1 in this host-parasite system, as every infection is lethal), $b$ is the rate of parasite independent mortality (assumed constant and negligible in our experiment), and $v$ is the host recovery rate (equal to zero here). Therefore, in our experiment, differences in parasite fitness across genetic and thermal variation could be fully attributed to changes in the transmission rate $\beta$, whose values were calculated by logistic regression of the data reflecting the proportion of infected filaments over physiological time. Parasite transmission-based fitness was hence expressed as transmission rate.

In addition, intensity of infection, size of sporangia and size of zoospores were evaluated.

184 Intensity of infection, or the mean number of infections (i.e. encysted zoospores or sporangia) 185 present on single hosts, was determined after examining 200 infected filaments per sample. Mean 186 sporangial volumes were estimated for 20 empty or mature sporangia per sample (distinguishable 187 by a well-developed thickened sporangium wall). Volume was estimated by measuring their two 188 semi-axes under a Nikon Ti Eclypse inverted microscope and the NIS-Element BR 4.5 software, and assimilating them as rotational ellipsoids with the volume

$$
\mathrm{V}=\pi / 6 * \mathrm{~d}_{1} 2 * \mathrm{~d}_{2}
$$

where $d_{1}$ and $d_{2}$ are the short and the long semi-axes, respectively. In case multiple empty/mature sporangia were present on a single host filament, only the biggest was measured and included in 
193 the analyses. In order to evaluate the potential effect of multiple infections on single hosts on

194 sporangial size (due to putatively increased competition for host resources), for every measured

195 empty/mature sporangium, the number of individual infections present on the host was also

196 recorded. Lastly, mean zoospore size was determined from the diameter of 50 measured

197 zoospores in each sample. Intensity of infection, mean sporangial volume and zoospore size were

198 determined from four subsamples per experimental unit (i.e. repeated measures) corresponding to

199 time points where the incidence of infection had plateaued. Thereby, a well-established level of

200 intensity of infection within the population, as well as a sufficient number of fully developed

201 sporangia and released zoospores were ensured.

203 Data analysis

204 Fixed effects of temperature and host strain were tested on each of the studied parasite fitness 205 traits: transmission rate, intensity of infection, mean volume of sporangia, and mean size of 206 zoospores. Transmission rate $(\beta)$ was calculated for each biological replicate by logistic 207 regression of data reflecting changes in incidence of infection over physiological time. Main and 208 interactive effects of host genotype and temperature on transmission rate were evaluated using a 209 linear model. This was followed by a contrast test comparing mean transmission rates within 210 individual host genotype treatments across temperatures, and between host genotypes within the same temperature (least-squares means test with Holm's p-value adjustment).

213 For intensity of infection, mean sporangial volume and zoospore sizes, fixed effects of 214 temperature, and host strain were examined by fitting linear mixed models. For sporangial 215 volume, data needed to be log-transformed to satisfy distributional assumptions of the residuals. 216 In order to account for repeated measures on biological replicates at four different time points 217 (see previous section), experimental unit (from which repeated measures were taken), and 218 experimental unit nested within sampling time were tested as possible random effects structures 219 in the respective models. However, regardless of the selected random factor structure, the 220 resulting estimates of variance components were zero, indicating that within-group variability 221 was not sufficient to warrant incorporating random effects in the models. In fact, including 222 random effects consistently resulted in poorer quality for the mixed models, as shown by higher 223 AIC values (Akaike Information Criterion, a relative measure of model quality), compared to 224 their non-mixed counterparts. The exclusion of random factors was further evaluated by fitting 
225 mixed and non-mixed models in parallel. In all cases, the results were almost identical and not 226 qualitatively different (data not shown). Therefore, the output of degenerate linear models is 227 reported, i.e. models where random effects were excluded, despite their initial inclusion as 228 imposed by the experimental design (Bates et al., 2015).

230 For all models, selection of optimal fixed covariate structures was performed by formulating full

231 models including all fixed terms (temperature, host strain, and their interaction term). Full models

232 were then compared with a nested model excluding one of the terms. Individual fixed terms were 233 excluded when such comparisons did not yield significant differences (i.e. including the term did 234 not significantly improve the model). After optimal model selection, the significance of 235 individual fixed terms was determined by Type III F-tests relative to an intercept-only model. In 236 addition, the proportion of variance explained by each fixed term is reported with regard to the 237 full model. This was calculated as the sequential sum of squares of each term, divided by the total 238 sum of squares in the full model. All statistical analyses were performed using Rstudio 239 (v.0.99.903). Linear mixed modeling was performed using the "lme4" package (Bates, 2010). 
Results

243 Cultures incubated at $8{ }^{\circ} \mathrm{C}$ did not become infected and were hence excluded from the analyses.

244 Incidence of infection increased fastest with physiological time at $20{ }^{\circ} \mathrm{C}$ compared to $16{ }^{\circ} \mathrm{C}$ and

$24512{ }^{\circ} \mathrm{C}$, reaching a plateau at around 100 degree-days (Figure 1a). Transmission rates were 246 accordingly higher at $20^{\circ} \mathrm{C}$, independently of the host strain infected (Figure 1b, Table 1). At 247 lower temperatures transmission rates dropped for both host strains. Most of the variance in the 248 data was explained by temperature (86.2\%, Table 1). Although host strain showed a significant 249 effect, it only explained about $7 \%$ of the variance. In fact, transmission rates did not display 250 significant differences between host strains, except at $12^{\circ} \mathrm{C}$ (Table 2). A significant host strain-

251 by-temperature interaction was found, although it displayed little explanatory power (3.1\%, Table $2521)$.

254 Intensity of infection (i.e. mean number of individual infections per infected host) on the NIVA255 CYA98 strain was conspicuously higher $(4.0 \pm 0.03)$ than on NIVA-CYA630 $(2.4 \pm 0.04)$, 256 regardless of temperature (Figure 2b). Host strain explained over $92 \%$ of the variance in the data, 257 whereas temperature did not have a significant effect in the model. A significant host strain-by258 temperature interaction was found, although it explained less than $1.5 \%$ of the variance of the 259 data (Table 1).

261 Mean sporangial volume was higher on NIVA-CYA98 than on NIVA-CYA630 (ca. 55\%). 262 Sporangial volumes decreased with increasing temperature (Figure 2a). In fact, linear models 263 showed that variation in sporangial volumes was mostly explained by both host strain and 264 temperature (34\% and 22\%, respectively; Table 1). A host strain-by-temperature interaction term 265 was maintained in the final model, although its effect on the mean volume of sporangia was 266 slightly below significance levels $(\mathrm{p}=0.051$; Table 1$)$.

Zoospore sizes decreased with increasing temperature independently of host genotype (Figure 2c). The reduced linear model showed that zoospore sizes were affected mostly by temperature (explaining over $66 \%$ of the variance), whereas host genotype only accounted for $9 \%$ of the variance (Table 1). The interaction term was not significant in the full model $\left(\mathrm{F}_{2,12} \mathrm{p}=0.186\right)$ and was excluded from the final model. 


\section{Discussion}

275 Overall chytrid fitness, as defined by transmission rate (May \& Anderson, 1983), was largely 276 modulated by thermal variation (Fig. 1a, Table 1). The chytrid parasite did not cause infections at

$2778{ }^{\circ} \mathrm{C}$, supporting the existence of a low-temperature infection refuge in this system and stressing 278 the importance of the external environment as a major modulator of chytrid disease outcome 279 (Gsell et al., 2013a; Rohrlack et al., 2015). The fact that host strain had only marginal 280 explanatory power on overall transmission-based fitness $(<7 \%$ of the variance; significant 281 differences among host strains were only found at $12{ }^{\circ} \mathrm{C}$ ) might initially point toward a limited 282 impact of host genotype on the outcome of infection. This, however, contrasts with earlier reports 283 of chytrid parasites of phytoplankton displaying rather narrow host ranges (Canter \& Jaworski, 284 1979; De Bruin et al., 2008; Sønstebø \& Rohrlack, 2011). While these discrepancies might be 285 initially attributed to the small set of host strains used here, which is unreflective of the natural 286 host genetic diversity, close examination of individual parasite traits did reveal significant effects 287 of host genotype on parasite performance. Intensity of infection (a measure of zoospore 288 encystment success) and size of sporangia and zoospores (jointly acting as proxies of parasite reproductive output) responded to host genotype and thermal variation in different ways, indicating that the interplay of these underlying traits jointly determines overall parasite fitness.

291 The parasite consistently performed better in all investigated individual traits when infecting the strain NIVA CYA98, most likely due to the fact that this strain was used for routine maintenance 293 of the parasite over years and the chytrid strain might hence have adapted to it (De Bruin et al., 2008). Still, the use of integrative fitness proxies (in this case transmission rates), masked these otherwise notorious differences among host strains observed when individual parasite traits were investigated. Differential response of individual parasite traits to environmental or host genotype variation are also common in other host-parasite systems (Fels \& Kaltz, 2006; Vale et al., 2008). Altogether, this stresses the important point that, by expressing parasite fitness in terms of transmission success, we integrate (but also overlook) underlying parasite traits that act as components of overall fitness, e.g. infection success, reproductive output, etc., into a single metric. Whereas integrative measures of fitness are often useful, they provide limited insights into the underlying mechanisms of infection. Instead, disentangling how individual parasite traits are affected by both host and external environment grants a deeper understanding of the ecophysiology of the host-parasite interaction. 
306 For instance, in contrast to overall fitness, intensity of infection was shown to be controlled 307 almost exclusively by host genotype, with temperature exerting no significant effect. This 308 supports the notion that chytrid encystment is likely mediated by cell-to-cell contact, with host 309 genotype-dependent cell surface characteristics acting as a first barrier against infection. By 310 analogy with other zoosporic parasites, cell surface features involved in chytrid-host 311 compatibility likely consist of carbohydrates and lectins that are prone to polymorphism across 312 conspecific strains (Hinch \& Clarke, 1980; Petre \& Kamoun, 2014). The fact that intensity of 313 infection (i.e. encystment success) was controlled by host genotype variation exclusively, 314 together with observations that the chytrid strain used here is able to encyst on some, but not all 315 conspecific host strains (Sønstebø \& Rohrlack, 2011), indicates that cell-to-cell compatibility 316 plays a pivotal role in defining host-parasite specificity in this system. Importantly, observed 317 differences in intensity of infection across susceptible host strains suggest that chytrid-host 318 compatibility at the cell surface level is not simply a binary trait (resistant/susceptible), but 319 encompasses different degrees of affinity among compatible host-parasite genotypes. Host 320 densities in our experiment were kept constant and equal encounter rates can hence be assumed 321 across treatments. Thus, observed changes in intensity of infection across host strains can be 322 solely attributed to dissimilar affinity of the parasite towards their respective cellular surfaces. 323 High cell-to-cell affinity might be a decisive factor for successful encystment and infection, especially under natural conditions where host densities (and hence parasite-host encounters) are 325 typically much lower than those provided in our experiment. Sporangial sizes also responded differently to host genotype and thermal variation compared to overall transmission-based fitness. Upon encystment, chytrids penetrate their host and secrete proteases to digest and extract nutrients from it. Therefore, final sporangial sizes arguably reflect the efficiency with which the parasite extracts and incorporates host resources into its own 331 biomass, ultimately determining its reproductive output. Exploitation efficiency can be 332 modulated, minimized, or suppressed by cyanobacterial hosts through the production of an array 333 of intracellular oligopeptides with diverse protease inhibiting properties (Welker \& von Dohren, 334 2006). Cyanobacterial knockout mutants unable to produce individual oligopeptides showed 335 increased susceptibility against a chytrid parasite, compared to the wild type strain (Rohrlack et 336 al., 2013), suggesting that these secondary metabolites are involved in anti-chytrid defense. 337 Interestingly, the synthesis of these cyanobacterial oligopeptides is genetically determined by the 
338 presence or absence of encoding gene clusters, whose distribution across conspecific 339 cyanobacterial strains in natural populations is remarkably patchy. This leads to chemically 340 polymorphic populations with potentially different susceptibility to a given chytrid parasite 341 (Agha \& Quesada, 2014). The host strains used in this experiment are no exception. Despite 342 small phylogenetic distance, they possess different intracellular oligopeptide compositions 343 (Rohrlack et al., 2008) that might lead to different susceptibility and thereby elicit differences in 344 final sporangial sizes under identical infection conditions. Beside host-specific defensive traits, 345 sporangial size might arguably be affected by the number of zoospores co-infecting a single host. 346 Co-infecting zoospores engage in competition for host resources and such competition might 347 modulate disease outcome. Indeed, an inverse relationship between the size of mature sporangia 348 and the number of infections present on the host was found (Fig. 3), supporting the idea that 349 increased competition among zoospores leads to reduced sporangial sizes. However, host 350 genotype differences in susceptibility seem to override this effect; in spite of a consistently higher 351 intensity of infection on strain NIVA-CYA98 (implying increased competition for host 352 resources), consistently bigger sporangia were found on this strain (Fig. 2). Moreover, for the 353 same number of infections, systematically bigger sporangia were found on strain NIVA-CYA98

354 (Fig. 3), further suggesting that host genotype differences in susceptibility override effects 355 derived from parasite intraspecific competition under multiple infections.

In light of the consistent positive correlations between sporangial size and the numbers of 358 contained zoospores repeatedly reported elsewhere (Bruning, 1991b; Gerphagnon et al., 2013; 359 Van den Wyngaert et al., 2014), sporangial size is typically regarded as a proxy of chytrid reproductive output. Therefore, we expected smaller sporangia under higher temperatures to imply a reduction in per capita reproductive output of the parasite. However, this contrasted with overall higher transmission rates observed at higher temperatures. Instead, together with a reduction in sporangial sizes, we also recorded a systematic reduction in zoospore sizes at higher temperatures. Chytrids might be able to compensate for reductions in final sporangial size by producing smaller zoospores, thereby stabilizing per capita reproductive output along temperature gradients. As zoospores rely on internal energy reserves to actively find a suitable host, this strategy might come at the cost of producing propagules with shorter infective lifetimes. However, shorter zoospore lifetimes might not impact parasite transmission when host densities are high, which typically coincides with the summer season and high temperatures. In addition to 
370 the production of smaller zoospores, high transmission rates at higher temperatures can also be

371 maintained by faster sporulation times (i.e. the time needed by the parasite to develop a fully 372 mature sporangium after encystment on the host; Van den Wyngaert et al., 2014), a fitness 373 component that we could not address with our experimental setup. Despite the obvious need to

374 demonstrate these possibilities, it is appealing to speculate that chytrids are able to exploit such 375 trade-offs between reproductive output and propagule longevity depending on environmental 376 context (e.g. host density, seasonal fluctuations) to maximize their fitness.

378 All in all, chytrid transmission success is the result of an interplay between individual traits, each 379 differently affected by the host and/or external environments. Successful evasion of host barrier 380 defenses (i.e. encystment success mediated by cell-to-cell compatibility) seems to be driven by

381 the host environment alone (genotype-by-genotype interactions). In contrast, parasite fitness traits 382 related to the efficiency with which the parasite extracts nutrients from the host appear to be 383 under joint control by both host and external environment, indicating genotype-by-environment 384 interactions. This exemplifies the importance of addressing underlying fitness traits to better 385 characterize the interaction between host and parasite, including infection mechanisms and the 386 influence of immediate and external environment on the outcome of the disease.

388 Financial support

389 This work was supported by a postdoctoral grant by the Alexander von Humboldt Foundation 390 granted to RA.

392 Acknowledgements

393 The authors would like to thank Mark Phillipo for proofreading the manuscript. Three

394 anonymous reviewers are also acknowledged for useful comments on an earlier version of the 395 manuscript. 
Agha, R. and Quesada, A. (2014). Oligopeptides as biomarkers of cyanobacterial subpopulations. Toward an understanding of their biological role. Toxins, 6, 1929-1950.

Agha, R., Saebelfeld, M., Manthey, C., Rohrlack, T. and Wolinska, J. (2016). Chytrid parasitism facilitates trophic transfer between bloom-forming cyanobacteria and zooplankton (Daphnia). Scientific Reports, 6.

404

405

406

Antolin, M. F. (2008). Unpacking $\beta$ : within-host dynamics and the evolutionary ecology of

407 pathogen transmission. Annual Review of Ecology, Evolution, and Systematics, 39, 415437.

408 preprint arXiv:1506.04967.

Bates, D. M. (2010). lme4: Mixed-effects modeling with R. Springer New York. gattung Rhizidium.

417 Bruning, K. (1991a). Effects of temperature and light on the population dynamics of the 
Bruning, K. (1991b). Infection of the diatom Asterionella by a chytrid. I. Effects of light on reproduction and infectivity of the parasite. Journal of Plankton Research, 13, 103-117.

423 Bruning, K. and Ringelberg, J. (1987). The influence of phosphorus limitation of the diatom Asterionella formosa on the zoospore production of its fungal parasite Rhizophydium planktonicum. Aquatic Ecology, 21, 49-54.

Canter, H. M. (1947). Studies on British Chytrids: II. Some new monocentric chytrids. Transactions of the British Mycological Society, 31, 94IN99-105IN110.

Canter, H. M. and Jaworski, G. (1979). The occurrence of a hypersensitive reaction in the planktonic diatom Asterionella formosa Hassall parasitized by the chytrid Rhizophydium planktonicum Canter emend., in culture. New Phytologist, 82, 187-206.

Canter, H. M. and Jaworski, G. (1981). The effect of light and darkness upon infection of Asterionella formosa Hassall by the chytrid Rhizophydium planktonicum Canter emend. Annals of Botany, 47, 13-30. 
444 De Bruin, A., Ibelings, B. W., Kagami, M., Mooij, W. M. and Van Donk, E. (2008). Adaptation of the fungal parasite Zygorhizidium planktonicum during 200 generations of growth on homogeneous and heterogeneous populations of its host, the diatom Asterionella formosa. Journal of Eukaryotic Microbiology, 55, 69-74. doi: 10.1111/j.1550-7408.2008.00306.x.

450 Falkowski, P. (2012). Ocean science: the power of plankton. Nature, 483, S17-S20.

451

Fels, D. and Kaltz, O. (2006). Temperature-dependent transmission and latency of Holospora undulata, a micronucleus-specific parasite of the ciliate Paramecium caudatum. Proceedings of the Royal Society of London B: Biological Sciences, 273, 1031-1038.

455

Frenken, T., Alacid, E., Berger, S. A., Bourne, E. C., Gerphagnon, M., Grossart, H. P., Gsell, A. S., Ibelings, B. W., Kagami, M. and Agha, R. (2017). Integrating chytrid fungal parasites into plankton ecology. Research gaps and needs. Environmental Microbiology, 19, 3802-3822.

460

Futuyma, D. J. (1986). Evolutionary biology, Sinauer, Sunderland, Mass.

462

Gerphagnon, M., Colombet, J., Latour, D. and Sime-Ngando, T. (2017). Spatial and temporal changes of parasitic chytrids of cyanobacteria. Scientific Reports, 7.

Gerphagnon, M., Latour, D., Colombet, J. and Sime-Ngando, T. (2013). A double staining method using SYTOX green and calcofluor white for studying fungal parasites of 
Grossart, H.-P., Wurzbacher, C., James, T. Y. and Kagami, M. (2016). Discovery of dark matter fungi in aquatic ecosystems demands a reappraisal of the phylogeny and ecology of zoosporic fungi. Fungal Ecology, 19, 28-38.

Gsell, A. S., de Senerpont Domis, L. N., Van Donk, E. and Ibelings, B. W. (2013a). Temperature alters host genotype-specific susceptibility to chytrid infection. PloS one, 8 , e71737.

Gsell, A. S., de Senerpont Domis, L. N., Verhoeven, K. J., Van Donk, E. and Ibelings, B. W. (2013b). Chytrid epidemics may increase genetic diversity of a diatom spring-bloom. The ISME Journal, 7, 2057-2059.

Hassett, B. and Gradinger, R. (2016). Chytrids dominate arctic marine fungal communities. Environmental Microbiology, 18, 2001-2009.

Hinch, J. M. and Clarke, A. E. (1980). Adhesion of fungal zoospores to root surfaces is mediated by carbohydrate determinants of the root slime. Physiological Plant Pathology, 16, 303IN301-307IN302. (2004). Host parasite interactions between freshwater phytoplankton and chytrid fungi (chytridiomycota) 1. Journal of Phycology, 40, 437-453. 
493 Kagami, M., von Elert, E., Ibelings, B. W., de Bruin, A. and Van Donk, E. (2007). The parasitic chytrid, Zygorhizidium, facilitates the growth of the cladoceran zooplankter, Daphnia, in cultures of the inedible alga, Asterionella. Proceedings of the Royal Society of London B: Biological Sciences, 274, 1561-1566.

498 Kraaijeveld, A. R. and Godfray, H. C. J. (1999). Geographic patterns in the evolution of resistance and virulence in Drosophila and its parasitoids. The American Naturalist, 153, S61-S74.

501

Lefèvre, E., Roussel, B., Amblard, C. and Sime-Ngando, T. (2008). The molecular diversity of freshwater picoeukaryotes reveals high occurrence of putative parasitoids in the plankton. PloS one, 3, e2324.

505

506 Lepère, C., Domaizon, I. and Debroas, D. (2008). Unexpected importance of potential parasites in the composition of the freshwater small-eukaryote community. Applied and Environmental Microbiology, 74, 2940-2949.

510 Lively, C. (1999). Migration, virulence, and the geographic mosaic of adaptation by parasites. The American Naturalist, 153, S34-S47.

513 May, R. M. and Anderson, R. M. (1979). Population biology of infectious diseases: Part II. Nature, 280, 455-461. 
516 May, R. M. and Anderson, R. t. (1983). Epidemiology and genetics in the coevolution of 517 parasites and hosts. Proceedings of the Royal Society of London B: Biological Sciences, $518 \quad 219,281-313$.

McCallum, H., Fenton, A., Hudson, P. J., Lee, B., Levick, B., Norman, R., Perkins, S. E., Viney, M., Wilson, A. J. and Lello, J. (2017). Breaking beta: deconstructing the parasite transmission function. Phil. Trans. R. Soc. B, 372, 20160084.

524 Mitchell, S. E., Rogers, E. S., Little, T. J. and Read, A. F. (2005). Host-parasite and genotype525 by-environment interactions: temperature modifies potential for selection by a sterilizing pathogen. Evolution, 59, 70-80.

Muehlstein, L. K., Amon, J. P. and Leffler, D. L. (1988). Chemotaxis in the marine fungus Rhizophydium littoreum. Applied and Environmental Microbiology, 54, 1668-1672.

Petre, B. and Kamoun, S. (2014). How do filamentous pathogens deliver effector proteins into plant cells? PLoS biology, 12, e1001801.

534 Rohrlack, T., Christiansen, G. and Kurmayer, R. (2013). Putative antiparasite defensive system involving ribosomal and nonribosomal oligopeptides in cyanobacteria of the genus Planktothrix. Applied and Environmental Microbiology, 79, 2642-2647.

538 Rohrlack, T., Edvardsen, B., Skulberg, R., Halstvedt, C. B., Utkilen, H. C., Ptacnik, R. and 539 Skulberg, O. M. (2008). Oligopeptide chemotypes of the toxic freshwater 

cyanobacterium Planktothrix can form subpopulations with dissimilar ecological traits. Limnology and Oceanography, 53, 1279.

543 Rohrlack, T., Haande, S., Molversmyr, Å. and Kyle, M. (2015). Environmental conditions 544 determine the course and outcome of phytoplankton chytridiomycosis. PloS one, 10, $545 \quad \mathrm{e} 0145559$.

547 Scholz, B., Küpper, F. C., Vyverman, W., Ólafsson, H. G. and Karsten, U. (2017). Chytridiomycosis of marine diatoms - The role of stress physiology and resistance in parasite-host recognition and accumulation of defense molecules. Marine Drugs, 15, 26.

Sommer, U., Adrian, R., De Senerpont Domis, L., Elser, J. J., Gaedke, U., Ibelings, B., Jeppesen, E., Lürling, M., Molinero, J. C. and Mooij, W. M. (2012). Beyond the Plankton Ecology Group (PEG) model: mechanisms driving plankton succession. Annual

Sønstebø, J. H. and Rohrlack, T. (2011). Possible implications of chytrid parasitism for population subdivision in freshwater cyanobacteria of the genus Planktothrix. Applied and Environmental Microbiology, 77, 1344-1351.

560 Vale, P. and Little, T. (2009). Measuring parasite fitness under genetic and thermal variation. Heredity, 103, 102.

563 Vale, P., Stjernman, M. and Little, T. (2008). Temperature-dependent costs of parasitism and 
maintenance of polymorphism under genotype-by-environment interactions. Journal of Evolutionary Biology, 21, 1418-1427.

566

567 Van den Wyngaert, S., Vanholsbeeck, O., Spaak, P. and Ibelings, B. W. (2014). Parasite

568 fitness traits under environmental variation: disentangling the roles of a chytrid's 569 immediate host and external environment. Microbial Ecology, 68, 645-656.

570

571 Vredenburg, V. T., Knapp, R. A., Tunstall, T. S. and Briggs, C. J. (2010). Dynamics of an emerging disease drive large-scale amphibian population extinctions. Proceedings of the National Academy of Sciences, 107, 9689-9694.

574

575 Welker, M. and von Dohren, H. (2006). Cyanobacterial peptides - Nature's own combinatorial 576 biosynthesis. FEMS Microbiology Reviews, 30, 530-563.

577

578 Wolinska, J. and King, K. C. (2009). Environment can alter selection in host-parasite 579 interactions. Trends in Parasitology, 25, 236-244. 
583 Figure 1. Change in incidence of infection with physiological time (A) and estimated 584 transmission rates (B) for different temperatures and host strains. (A) Lines represent logistic fits 585 of data from pooling all three biological replicates. (B) Transmission rates ( $\beta$ ) were estimated 586 from logistic regressions for each biological replicate
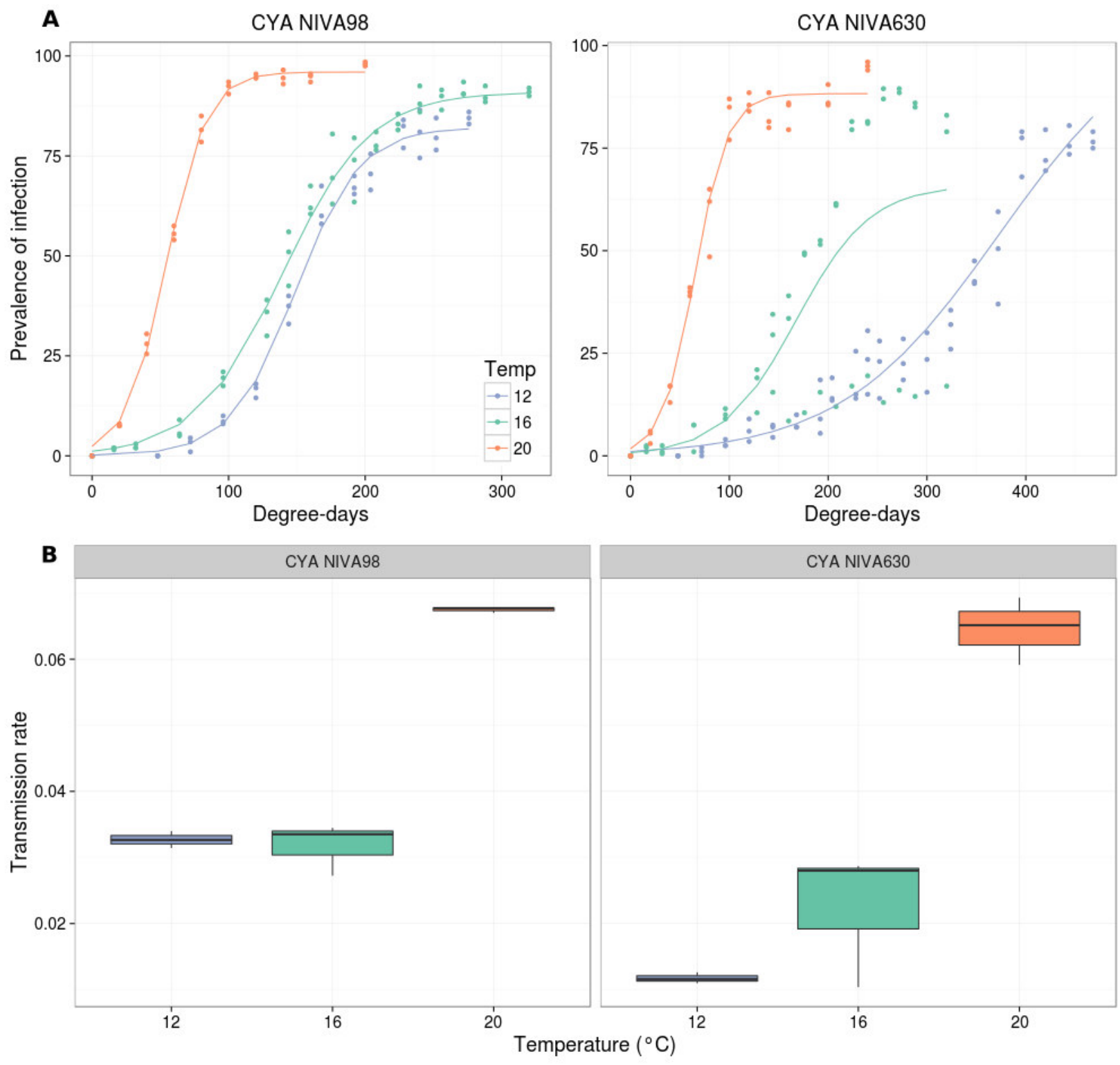
591 Figure 2. Mean sporangial biovolume, intensity of infection and zoospore sizes $( \pm$ SE) under 592 different host strain and temperature combinations
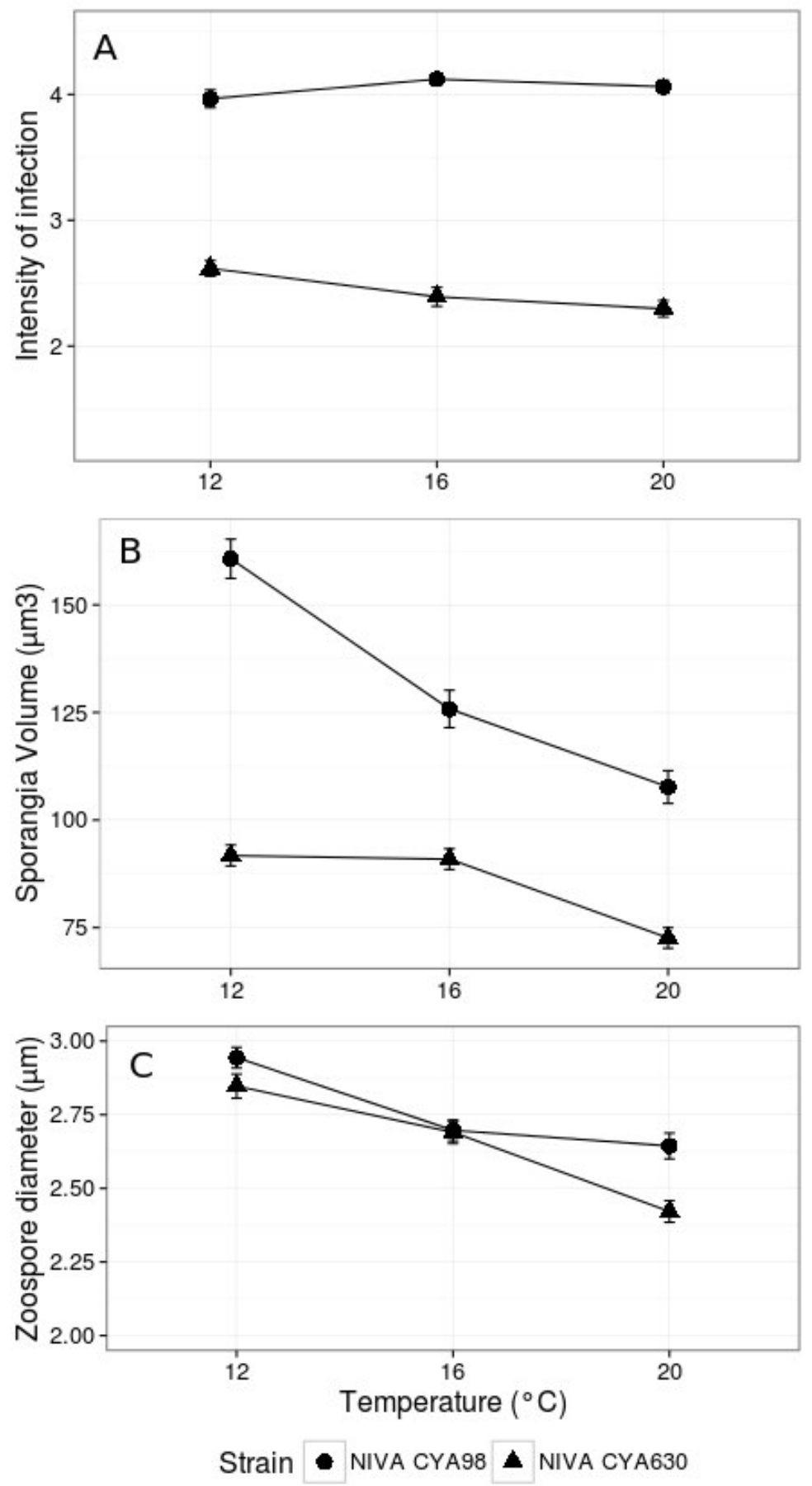

593

594

595 
596 Figure 3. Relation between the number of infections on a single host and the volume of the 597 biggest mature sporangium at the tested temperatures. Data represent 80 sporangial

598 measurements per temperature - host strain combination.

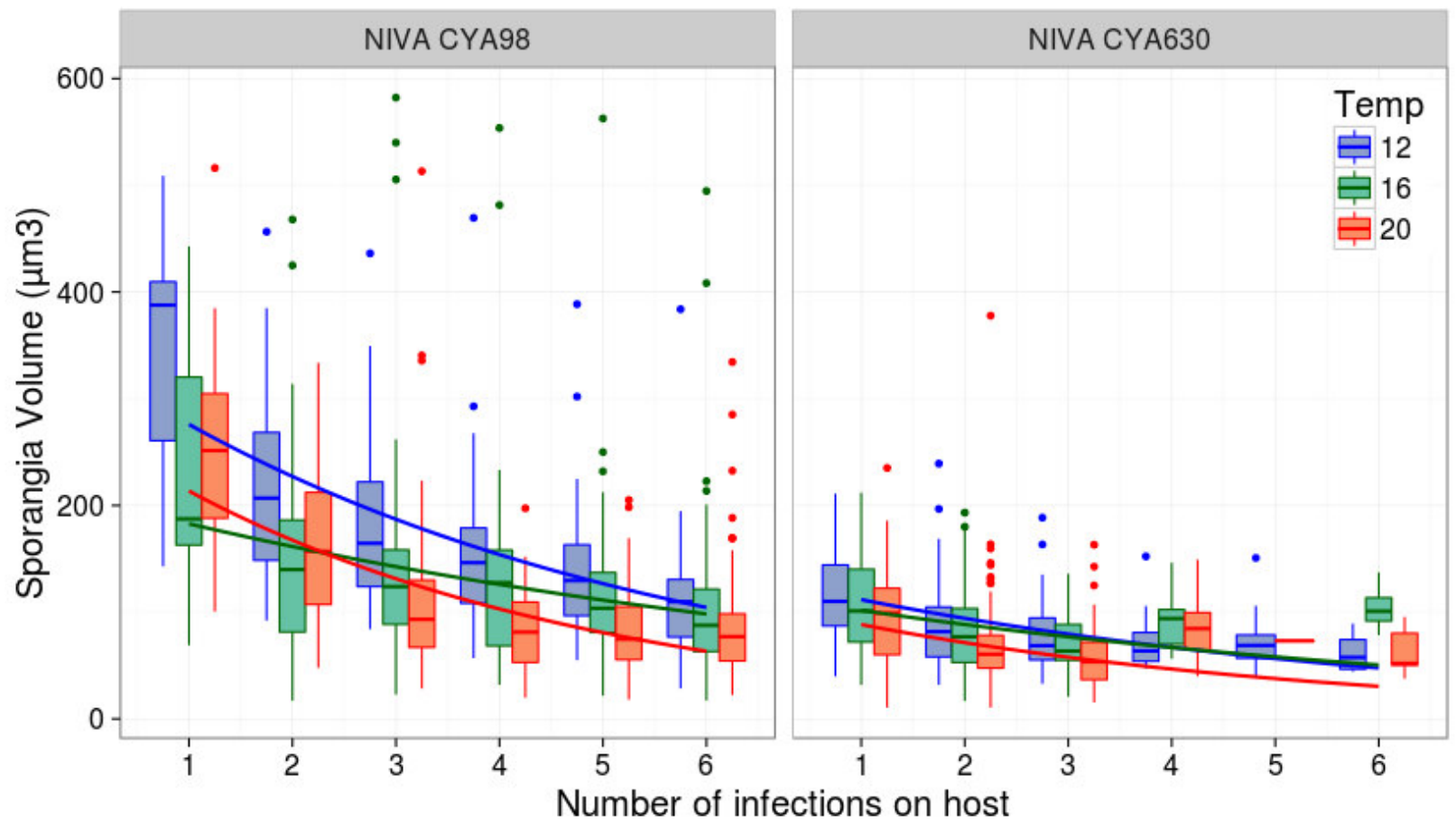

599

600 
601 Table 1. Linear models for fixed effects of host strain, temperature, and their interaction on 602 parasite transmission rates, intensity of infection, sporangial volumes and zoospore sizes. For 603 zoospore sizes, the interaction term was removed in the reduced model as its inclusion did not 604 significantly improve the full model. Variance explained on individual terms stems from sum 605 squares quotients in the full model. df: degrees of freedom; NS: Not significant (i.e. term was 606 excluded in the reduced model)

607

608

\begin{tabular}{|c|c|c|c|c|c|}
\hline \multirow[b]{2}{*}{ Transmission rate $(\beta)$} & \multicolumn{3}{|c|}{ Reduced model } & \multicolumn{2}{|c|}{ Full model } \\
\hline & & & & & \\
\hline & $\mathrm{df}$ & F ratio & $\operatorname{Pr}>F$ & $\mathrm{df}$ & $\% \mathrm{Vi}$ \\
\hline Host Strain & 1 & 21.89 & 0.0005 & 1 & 6.9 \\
\hline Temperature & 2 & 136.56 & $<0.0001$ & 2 & 86.2 \\
\hline Host Strain $\times$ Temperature & 2 & 4.93 & 0.0274 & 2 & 3.1 \\
\hline Residuals & 12 & & & 12 & 3.8 \\
\hline Intensity of infection & & & & & \\
\hline Host Strain & 1 & 995.53 & $<0.0001$ & 1 & 92.3 \\
\hline Temperature & 2 & 1.68 & 0.1949 & 2 & 0.3 \\
\hline Host Strain $\times$ Temperature & 2 & 6.80 & 0.0021 & 2 & 1.3 \\
\hline Residuals & 12 & & & 12 & 6.1 \\
\hline
\end{tabular}

Sporangia volume

$\begin{array}{llllll}\text { Host Strain } & 1 & 61.76 & <0.0001 & 1 & 35.1 \\ \text { Temperature } & 2 & 21.03 & <0.0001 & 2 & 23.9 \\ \text { Host Strain } \times \text { Temperature } & 2 & 3.12 & 0.051 & 2 & 3.5 \\ \text { Residuals } & 12 & & & 12 & 37.5\end{array}$

Zoospore size

$\begin{array}{llllll}\text { Host Strain } & 1 & 5.17 & 0.039 & 1 & 9.0 \\ \text { Temperature } & 2 & 19.23 & <0.001 & 2 & 66.7 \\ \text { Host Strain } \times \text { Temperature } & - & \text { NS } & \text { NS } & 2 & 5.9 \\ \text { Residuals } & 14 & & & 12 & 18.4\end{array}$


612 Table 2. Contrast tests for transmission rates. Comparisons are made for infections on the same 613 host strain under different temperatures, and for different host strains under the same temperature. 614 Significant p-values are depicted in bold.

615

616

617

Transmission rate

(B)

\begin{tabular}{|c|c|c|c|c|c|c|}
\hline & & \\
\hline & & $16^{\circ} \mathrm{C}$ & $20^{\circ} \mathrm{C}$ & $12^{\circ} \mathrm{C}$ & $16^{\circ} \mathrm{C}$ & $20^{\circ} \mathrm{C}$ \\
\hline \multirow{3}{*}{ NIVA CYA98 } & $12^{\circ} \mathrm{C}$ & 0.9998 & $<0.0001$ & 0.0027 & - & - \\
\hline & $16^{\circ} \mathrm{C}$ & - & $<0.0001$ & - & 0.2717 & - \\
\hline & $20^{\circ} \mathrm{C}$ & - & - & - & - & 0.9757 \\
\hline \multirow{2}{*}{ NIVA CYA630 } & $12^{\circ} \mathrm{C}$ & - & - & - & 0.1742 & $<0.0001$ \\
\hline & $16^{\circ} \mathrm{C}$ & - & - & - & - & $<0.0001$ \\
\hline
\end{tabular}

618 